\title{
A Low Power Clocked Integrated-and-Fire Modulator for UWB Applications
}

\author{
Iman Kianpour, Bilal Hussain, Vitor G. Tavares, Helio S. Mendonca \\ INESC-TEC and Faculty of Engineering of the University of Porto (FEUP) \\ Porto, Portugal \\ \{ikian, bhussain,vgt, hsm\}@fe.up.pt
}

\begin{abstract}
An integrate-and-fire modulator (IFM) is designed for power scavenging systems like: Wireless Sensor Network (WSN) and Radio Frequency Identification (RFID) sensor tags. The circuit works with a clock in order to be able to be synchronized with microprocessors, which must be used to reconstruct the signal. The modulator is simulated using $130 \mathrm{~nm}$ CMOS technology and the resulting power consumption is around $14 \mathrm{nW}$ at a clock frequency of $10 \mathrm{kHz}$. The OTA individually dissipates roughly $13 \mathrm{nW}$. Signal reconstruction resulted in a 9.2 ENOB.
\end{abstract}

Index Terms-- Integrate-and-fire neurons, Low power design, UWB transmission, low power design

\section{INTRODUCTION}

Neurons are diverse in terms of functionality and behavior. Among different models, integration-and-fire neuron (IFN) is a simplified version that is reduced to a first order fire-andreset system [1]. In this view, the aggregated charge from external excitation raises the neuron's cell-membrane potential until it reaches a threshold. Then, the neuron generates a spike and the membrane potential resets to a reference value and again starts accepting inputs from neighboring neurons. Basically, the membrane can be thought as a capacitor that relates the membrane potential (action potential) with the excitation current [1]. The resulting output pulse-density (firing rate) is proportional to the net input excitation. This process, also known as Pulse Density Modulation (PDM), converts the analog input signal to a series of time-encoded spikes, profiting from a dynamic sampling rate [1], [2]. In other words, its function is time encoding that maps the amplitude information, of an arbitrary stimulus, to a series of time distances $(\delta)$ between fixed- and short-duration spikes (Fig. 1) [3].

In power scavenging systems like WSN and RFID tags, there is a huge effort to design low-power analog-to-digital converters (ADC) or even sometimes to eliminate it from the transmitter block diagram [4]. Pulse width modulation (PWM) or integrate-and-fire modulation, which is also known as IFN modulator or IFN converter, could be utilized as an ADC replacement [4], [5] and at the same time be regarded as actual encode/modulation scheme. The integrate-and-fire modulation technique can then be used in Impulse Radio Ultra Wide-Band (IR-UWB) transmitters. A study on power efficiency of this transmitter shows that it roughly dissipates 8 times less power than a digital BPSK transmitter [6].

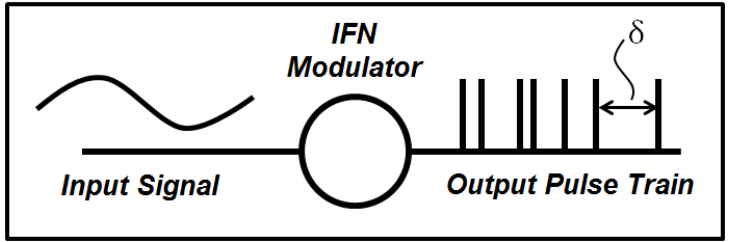

Figure 1. Function of integrate-and-fire neuron as a modulator

In this paper, a low power clocked-based integrate-and-fire modulator has been designed for RFID and WSN systems involving slow varying signals. The rest of this paper is organized as follows: in section II, the circuitry of the modulator will be discussed. In section III, the reconstruction method is completely described. Finally, results have been presented in section IV, followed by a conclusion in section $\mathrm{V}$.

\section{CIRCUITRY OF INTEGRATE-AND-FIRE MODUlATOR}

An integrate-and-fire modulator (IFM) is composed of an integrator, a comparator and a delay circuit as illustrated in Fig. 2. First, the input signal is integrated in time and then compared with a reference voltage $(\theta)$, this will be carried out every $1 / f_{S}$ second. As soon as the integration reaches the threshold $\theta$, the comparator activates the switch to reset the integrator. However, this happens after a certain amount of time $\tau_{r}$, called refractory period generated by a delay circuit, which can be realized by an asymmetric inverter with controllable current on the falling edge. The response for the integrator can be represented as [3]:

$$
\int_{t_{i}+\tau_{r}}^{t_{i+1}} x(t) d t=\theta, \quad \forall i \in \mathbb{Z}
$$

where $x(t)$ is the input signal and $t_{i}(i \in \mathbb{Z})$ is a timing sequence. The minimum sample rate in IFM is related to maximum adjacent interval $\left(\delta_{\max }=\sup \left(t_{i+1}-t_{i}\right)\right)$.

This work is sponsored by European Union (EU) under ITN FP7 program for a project named SENSEIVER (Low cost and energy-efficient LTCC sensor/IR-UWB transceiver solutions for sustainable healthy environment) with grant agreement number: 289481. 
Obviously, the input signal can be perfectly recovered provided that the minimum sample rate is above Nyquist rate, or that is to say $\delta_{\max }<1 / f_{\text {Nyquist }}$ [3], [7].

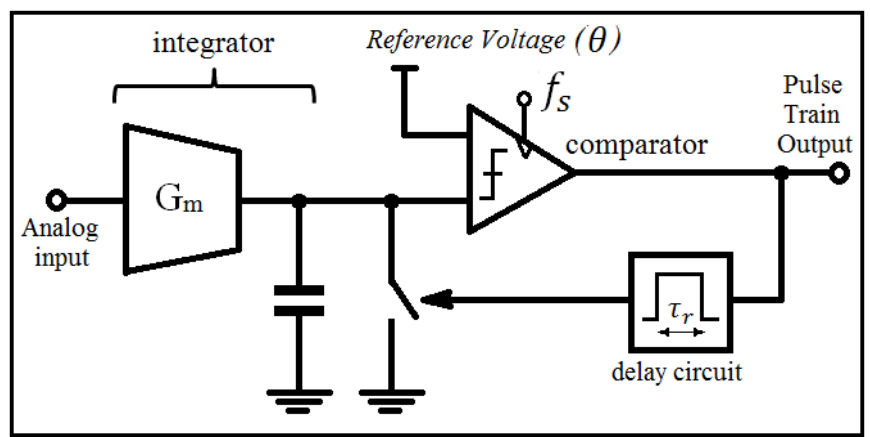

Figure 2. Architecture of the integrate-and-fire modulator

The IFN converter can serve as a baseband modulator in a UWB transmitter. IR-UWB pulses can be used to actually send the IFN spikes. Let us call this communication system Impulse-Radio Integrate-and-Fire (IRIF) transceiver. The IRIF transmitter comprises an IFM and an impulse-radio generator that will send enough number of UWB pulses representing each IFN pulse. The construction of the transmitting signal based on IFN spikes is shown in Fig. 3. Since the pulse generator is deactivated most of the time, power efficiency will greatly improve [6].

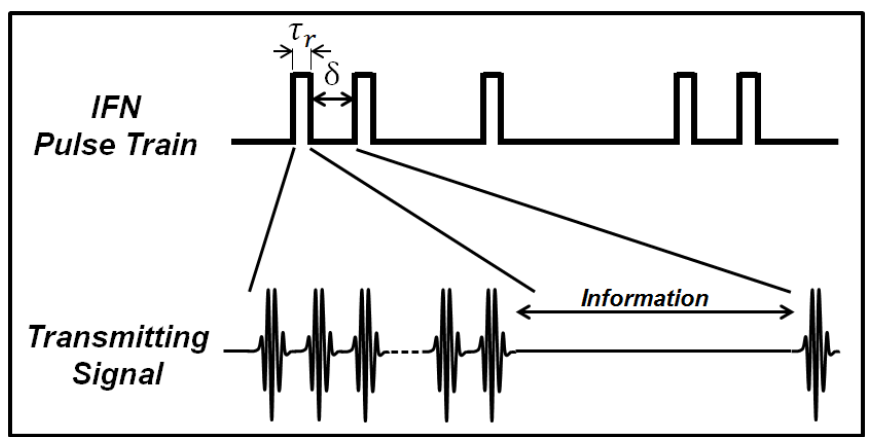

Figure 3. To generate transmitting signal based on output signal of the IFM. A series of UWB pulses represent each IFN pulse.

Sampling rate in conventional ADCs is constant, whereas IFM enjoys a dynamic sampling rate, which depends on the shape of the input signal and it may be either above or at the Nyquist rate [2]. This dynamic sampling rate is inherently non-uniform, leading to significantly better spectrum efficiency. Moreover, by using integrate-and-fire time encoding, lower power consumption is achievable.

\section{A. Gm-C Integrator}

An Operational Transconductance Amplifier (OTA) is designed in weak inversion region of operation using a multitanh triplet [8]. Multi-tanh topology is chosen to increase the linear input range and its power dissipation is reduced by taking the advantage of designing it in weak inversion. The OTA uses a composite transistor to degenerate the differential pairs, gaining a very small third harmonic distortion (HD3) [8]. The transconductance value is $37 \mathrm{nS}$, with a linear range of $340 \mathrm{mVpp}$, dissipating power of almost $13 \mathrm{nW}$. OTA is the major power consumer circuit block of the whole IFM.

\section{B. Comparator}

Latch-type sense amplifiers, or sense amplifier based flipflops are kind of track-and-latch $(\mathrm{T} / \mathrm{L})$ comparators and are widely used in memories, ADCs and on-chip transceivers [9]. They achieve fast decisions due to a strong positive feedback. Since in most practical applications IFN action potentials should be synchronized to a fast clock of a processor, a low power $\mathrm{T} / \mathrm{L}$ comparator is used in this modulator [10]. It has no static current and therefore suitable for low power circuit. The comparator dissipates roughly $1 \mathrm{nW}$ of power at clock frequency of $10 \mathrm{kHz}$. Its comparison delay is $25 \mathrm{ps}$ and the offset voltage at the input is roughly $10 \mathrm{mV}$.

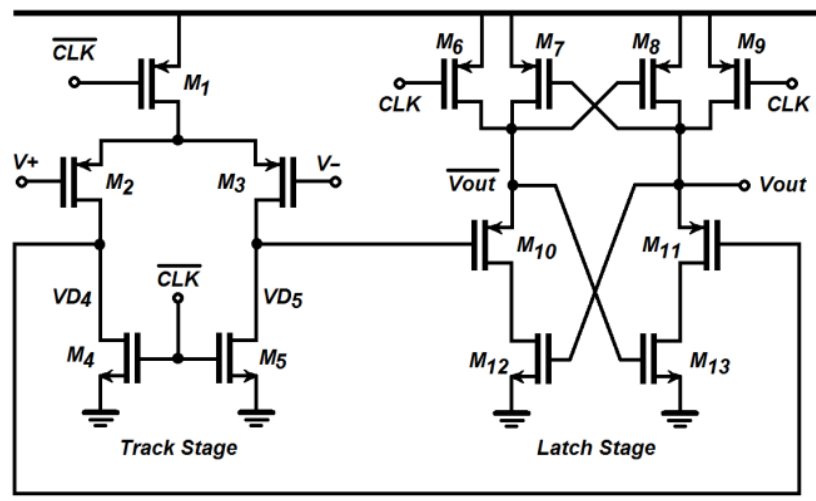

Figure 4. Track-and-Latch comparator

The comparator is composed of two stages, namely track stage and latch stage. The first stage instantaneously tracks the difference between input terminals. The latch stage will store the comparison results. In detail, the comparator functions as follows. First, the clock level is low and because the channels of M4 and M5 are inverted, the nodes VD4 and VD5 are precharged "low"; meanwhile $\mathrm{V}_{\text {out }}$ and $\overline{\mathrm{V}_{\text {out }}}$ are pre-charged "high", and M6 and M9 are on. In a rising clock edge current flows through M1 and charge the intrinsic capacitors in nodes VD4 and VD5. Depending on input voltages and their difference, one of the capacitors will charge faster than the other, turning on either M10 or M11. As long as the clock is high the data is latched at the output.

\section{Delay Circuit}

The delay circuit is responsible for providing enough refractory time letting the capacitor discharge completely. The circuit can be easily realized as shown in Fig. 5. It is basically an inverter with a delay in the falling edge, which is generated through current starvation controlled by a MOSFET. By using the current controller, the falling edge becomes smoother and, therefore, the last two inverters seen in Fig.5 are utilized to provide correct logic and create a sharp transition at the output. 


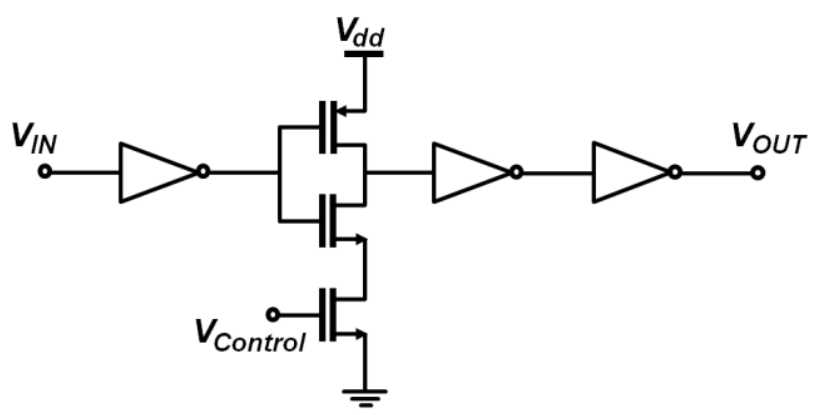

Figure 5. Delay circuit: a current controller inverter

\section{Signal Reconstruction Method}

Several approaches can be adopted to reconstruct the original signal based on the received spikes, either in timedomain [7], [11] or frequency-domain [12]. However, a simple recovery algorithm is used [7] to verify the performance of the modulator, which has been carried out by MATLAB. Since any band-limited signal can be expressed as a low-pass filtered version of an appropriately weighted sum of delayed impulse functions [10], [13], for an $\Omega_{\mathrm{s}}$ bandlimited signal $\mathrm{x}(\mathrm{t})$, which has $\mathrm{k}(\mathrm{k} \in \mathbb{Z})$ IFN spikes within length of $l(l \in \mathbb{Z})$, equation (2) can be used to decompose the signal into spike trains.

$$
\begin{aligned}
x(t)=h(t) * \sum_{j=1}^{k} \omega_{j} \delta\left(t-s_{j}\right) \\
=\sum_{j=1}^{k} \omega_{j} h\left(t-s_{j}\right)
\end{aligned}
$$

In equation (2), $s_{j}=\left(t_{j+1}+t_{j}\right) / 2, \omega_{j}=x\left(s_{j}\right)$ and $h(t)$ is the impulse response of the ideal low-pass filter represented in equation (3) and ' $*$ ' represents the convolution operator.

$$
h(t)=\frac{\sin \Omega_{s} t}{\pi t}
$$

Denoting by $[q]_{l}=x\left(t_{l}\right)$ and the vector of weights $[W]_{k}=\left[\omega_{j}\right]$, equation (2) can be rewritten in a matrix form as follows:

$$
q=H^{T} W
$$

where $T$ denotes the transpose and $[H]_{k, l}=\left[h\left(t-s_{j}\right)\right]$ is a matrix where each row gives the vector of an $s_{j}$-shifted impulse response of the ideal low-pass filter with bandwidth slightly bigger than $\Omega_{s}$. If the weights are known, the signal can be recovered. Substituting equation (2) into equation (1), we obtain:

$$
\theta_{i}=\sum_{j} \omega_{j} \int_{t_{i}}^{t_{i+1}} h\left(t-s_{j}\right) d t=\sum_{j} \omega_{j} c_{i, j}
$$

where $c_{i, j}$ are coefficients of a matrix where its columns give the values of integrals for each $s_{j}$-shifted sinc over all $\left[t_{i}, t_{i+1}\right]$ and can be numerically calculated from the following equation.

$$
c_{i, j}=\int_{t_{i}}^{t_{i+1}} h\left(t-s_{j}\right) d t
$$

So, the weights are simply computed by $\boldsymbol{W}=\boldsymbol{C}^{-1} \boldsymbol{\theta}$ and finally using equation (4), the input signal can be reconstructed as follows:

$$
\boldsymbol{q}=\boldsymbol{H}^{T} \boldsymbol{C}^{-1} \boldsymbol{\theta}
$$

\section{Simulation RESUlts AND Discussion}

The proposed clocked based IFM is designed in the 0.13 um technology node with $1.2 \mathrm{~V}$ power supply (technology voltage). The total average power consumed by the modulator is around $14 \mathrm{nW}$ at $10 \mathrm{kHz}$ clock frequency, and shows a linear dependency on frequency. In some recent publications, the power dissipation has not been considered as a major concern [7]; however, CMOS-based IFN modulators with $100 \mathrm{uW}$ and $300 \mathrm{nW}$ power consumptions are respectively reported in [11] and [15].

Figure 6 illustrates how the signal is recovered using a clock frequency of $100 \mathrm{kHz}$ applied to the comparator, while the reference voltage is set to $100 \mathrm{mv}$. In order to test the circuit, a sine wave with frequency of $20 \mathrm{~Hz}$ has been applied to the circuit, which can be seen in Fig. 6-a. Time encoded pulse train generated by IFM is illustrated in Fig. 6-b. With $100 \mathrm{mv}$ reference voltage, around 25-to-30 IFN spikes will be created in each period of the input sine wave. Figure 6-c shows the reconstructed signal. The Signal to Error power Ratio (SER) is $57 \mathrm{~dB}$ and ENOB of 9.2 has been achieved. The error is demonstrated in Fig. 6-d, which is the difference between input signal and the reconstructed one. As the clock frequency increases, the time resolution between spikes will increase so that the reconstruction quality will improve and higher ENOB is achievable.

Quality of the signal reconstruction and more details are given in TABLE I for the case of Vref $=100 \mathrm{mv}$. The quality of the reconstruction of the signal also depends on the harmonic distortion of the OTA and the precision of the reconstruction algorithm. In order to obtain an ENOB of 9.2 almost 28 IFN spikes are required. However, a digital BPSK transmitter needs to send 368 bits to reach the same ENOB as IR-IF transmitter. Therefore, the proposed communication system 
consumes less power comparing to an ordinary BPSK transmitter.

(a)

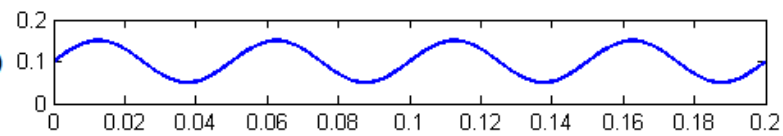

(b)

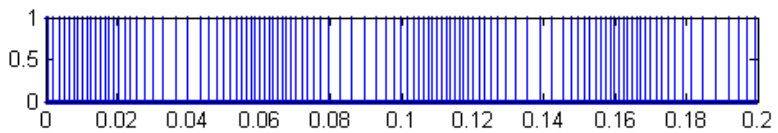

(c)

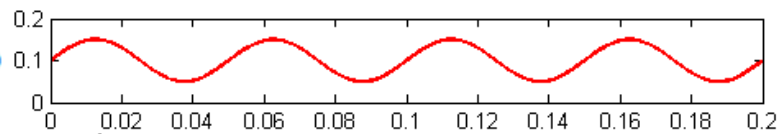
$\times 10^{-3}$

(d)

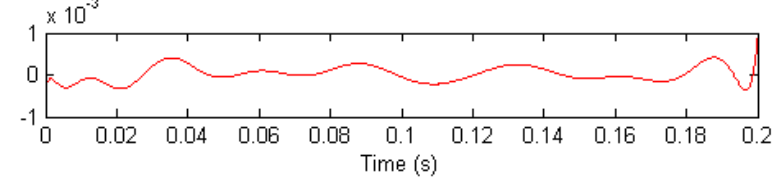

Figure 6. Signal reconstruction. (a) Input signal: a 20Hz sine wave. (b) Integrate-and-Fire pulse trains generated based on input signal using IFM. (c) Reconstructed signal. (d) Error between the original and reconstructed signal.

TABLE I. TABLE TyPe Styles

\begin{tabular}{|c|c|c|c|}
\hline $\begin{array}{c}\text { Clock } \\
\text { Frequency }\end{array}$ & ENOB & SER & $\begin{array}{c}\text { Spike per } \\
\text { period }\end{array}$ \\
\hline $10 \mathrm{KHz}$ & 5.1 & $32 \mathrm{~dB}$ & 26 \\
\hline $100 \mathrm{KHz}$ & 9.2 & $57 \mathrm{~dB}$ & 28 \\
\hline
\end{tabular}

\section{CONCLUSION}

A clocked-based monophasic IFM has been designed in 0.13 um technology. This modulator can be used in WSN, RFID sensor tag applications and generally in those systems dealing with slow varying signals. The results show that the power consumption of the whole modulator is as low as $14 \mathrm{nW}$ at $1.2 \mathrm{~V}$. Using MATLAB signal has been recovered perfectly with power ratio of $\mathrm{SER}=57 \mathrm{~dB}$ and $\mathrm{ENOB}$ of 9.2 .

\section{REFERENCES}

[1] W. Gerstner and W. . M. Kistler, , Spiking Neuron Models: Single Neurons, Populations, Plasticity, Cambridge: Cambridge University Press, 2002

[2] H. G. Feichtinger and K. Gröchenig, Theory and practice of irregular sampling, in Wavelets: Mathematics and Applications, Boca Raton, FL: CRC Press, Studies in Advanced Mathematics, 1994, pp. 305-363.
[3] A. A. Lazar, "Time encoding withan integrate-and-fire neuron with a refractory period," Elsevier, p. 53 - 58, 2003.

[4] M. Ghovanloo and M. Yin, "A clockless ultra low-noise low-power wireless implantable neural recording system," in IEEE International Symposium on Circuits and Systems (ISCAS), 2008.

[5] M. Rastogi, A. S. S. Alvarado, J. G. Harris and J. C. Principe, "Integrate and fire circuit as an ADC replacement," in IEEE International Symposium on Circuits and Systems (ISCAS), Rio de Janeiro, 2011.

[6] I. Kianpour, B. Hussain, V. G. Tavares, C. Duarte, H. S. Mendonca and J. Principe, "An Energy Study on IR-UWB Transmitter Using Integration-and-Fire Modulation," in IEEE International Conference on Ultra-Wideband (ICUWB), Paris, 2014.

[7] D. Wei and J. Harris, "Signal reconstruction from spiking neuron models," in IEEE International Symposium on Circuits and Systems (ISCAS), 2004.

[8] I. Kianpour, B. Hussain, V. G. Tavares and H. S. Mendonca, "A LowPower Multi-Tanh OTA with Very Low Harmonic Distortion," in International Symposum on Circuit and System (ISCAS), Lisbon, 2015.

[9] D. Schinkel, E. Mensink, E. A. M. Klumperink, E. v. Tuijl and B. Nauta, "A 3-Gb/s/ch transceiver for 10-mm uninterrupted RC-limited global on-chip interconnects," IEEE Journal of Solid-State Circuits, vol. 41, no. 1, pp. 297-306, 2006.

[10] D. Schinkel, E. Mensink, E. Klumperink, E. V. Tuijl and B. Nauta, "A Double-Tail Latch-Type Voltage Sense Amplifier with 18ps Setup+Hold Time," in Solid-State Circuits Conference (ISSCC), San Francisco, CA, 2007.

[11] D. Chen, Y. Li and D. Xu, "Asynchronous Biphasic Pulse Signal Coding and its CMOS Realization," in IEEE, International Symposium on Circuits and Systems (ISCAS), 2006.

[12] A. Alvarado, J. Principe and J. Harris, "Stimulus reconstruction from the biphasic integrate-and-fire sampler," in 4th International IEEE/EMBS Conference on Neural Engineering, Antalya, 2009.

[13] D. Wei and J. Harris, "Signal reconstruction from spiking neuron models," in International Symposium on Circuits and Systems, 2004.

[14] H. Feichtinger and K. Grochenig, "Theory and practice of irregular sampling," in in J.J. Benedetto and M.W. Frazier, editors, Wavelets: Mathematics and Applications, Boca Raton, FL, 1994.

[15] G. Indiveri, "A Low-Power Adaptive Integrate-and-Fire Neuron Circuit," in IEEE International Symposium on Circuits and Systems, (ISCAS), 2003. 